\title{
The Efficient Oxygen Reduction Catalysts Based on the Non- Noble Metal and Conducting Polymers
}

\author{
Tingting Yang ${ }^{1}$, Min $\mathrm{Wang}^{2}$, Xiuping Ju $\mathrm{u}^{3}$, Jinsheng Zhao ${ }^{1, *}$, Chonggang $\mathrm{Fu}{ }^{1, *}$ \\ ${ }^{1}$ Department of Chemistry, Liaocheng University, Liaocheng, 252059, P.R. China. \\ ${ }^{2}$ Liaocheng People's Hospital, Liaocheng, 252000, P.R. China. \\ ${ }^{3}$ Dongchang College, Liaocheng University, Liaocheng, 252059, P.R. China. \\ *E-mail: j.s.zhao@163.com (j.s.zhao); cgfu@lcu.edu.cn (c.g.Fu)
}

doi: $10.20964 / 2017.12 .108$

Received: 15 October 2017 / Accepted: 10 November 2017 / Published: 12 November 2017

\begin{abstract}
This paper describes the synthesis and assessment of a non-noble-metal electrocatalyst for oxygen reduction reaction (ORR). The transition-metal-coordinating nitrogen-doped carbon catalyst (M-N/C) is prepared by supporting copper ion on carbon-coated poly(2,7-dimethyl-10,12-di(thiophen-2yl)thieno[3',4':5,6]pyrazino[2,3-f][1,10]phenanthroline) (PDTPP) complex to offer Cu-PDTPP/C/GC. The materials including PDTPP, PDTPP/C, and $\mathrm{Cu}$-PDTPP/C are characterized by $\mathrm{CV}$ (cyclic voltammetry), RDE (rotating disk electrode) to confirm their catalytic performances. Among the four materials, Cu-PDTPP/C shows the best catalytic performance with the direct reduction of oxygen to water directly by a four-electron (4e) transferred process. The morphologies and chemical compositions of PDTPP/C, Cu-PDTPP/C were characterized by scanning electron microscopy (SEM) and X-ray photoelectron spectroscopy (XPS) methods respectively. it is revealed by the results that the copper ions have been coordinated to the composite and the $\mathrm{M}-\mathrm{N} / \mathrm{C}$ catalysts are successfully synthesized. According to these tests, this study essentially illustrate that $\mathrm{Cu}-\mathrm{PDTPP} / \mathrm{C}$ is an effective catalyst can be used for ORR in fuel cells, such as microbial fuel cells, which conducted in a aqueous neutral medium.
\end{abstract}

Keywords: Oxygen reduction reaction; M-N-C electrocatalyst; Polymerization

\section{$\underline{\text { FULL TEXT }}$}

(C) 2017 The Authors. Published by ESG (www.electrochemsci.org). This article is an open access article distributed under the terms and conditions of the Creative Commons Attribution license (http://creativecommons.org/licenses/by/4.0/). 